\title{
Estrategias que potencializan la lectura en el aprendizaje del idioma inglés
}

\author{
Strategies to improve the reading skill in the English language learning
}

\author{
Leticia Mercedes Cedeño Macías*, y Ángela Rosario Flores Vélez ${ }^{\dagger}$ \\ Universidad Técnica de Manabí, Ecuador. \\ \{lmcedeno,aflores\}@utm.edu.ec
}

Fecha de recepción: 12 de diciembre de 2015 - Fecha de aceptación: 15 de febrero de 2016

\begin{abstract}
Resumen-La lectura es una de las destrezas comunicativas de Inglés como lengua extranjera, y por lo tanto es un tema de estudio en el proceso de enseñanza aprendizaje de este idioma, es una destreza receptiva y no suele ser muy popular entre los estudiantes de lenguas por considerarla pasiva, monótona e incluso aburrida, todo lo cual se contrapone con la naturaleza extrovertida y dinámica de los jóvenes del siglo XXI. Pero ¿es realmente la lectura una actividad pasiva? El objetivo de este estudio es demostrar que la lectura puede ser una actividad participativa en la que los estudiantes puedan interactuar en parejas o en grupos y que fomente la comunicación a través de estrategias activas y participativas puntuales como el mapa mental, el debate, la rejilla y el panel, las cuales potencializan la lectura a través de una correcta y eficaz planificación del docente. La metodología que se utilizará será la revisión bibliográfica mediante el análisis de textos científicos y la experiencia de expertos en la enseñanza de Inglés como lengua extranjera.
\end{abstract}

Palabras Clave-Estrategias activas y participativas, Comprensión lectora, Mapa mental, Debate, Rejilla, Panel.

Abstract-Reading is one of communicative skills, so it is one of the topics all English learners have to study, it is a receptive skill and it is not using to be very popular among language students because they think it is passive, monotonous and even boring, and all these characteristics are against the extrovert and dynamic nature of youth who live in this century. But, is reading really a passive activity? The objective of this essay is to demonstrate that reading is a participative activity where students can interact in pairs or groups improving the communication through certain active and participative strategies like mind map, debates, drills and panels throughout an effective teacher's planning. The methodology used for this study will be the bibliographic over the analysis of English teaching experts' books and their experience.

Keywords-Active and participative strategies, Reading comprehension, Mind map, Debate, Drills, Panel.

\section{INTRODUCCIÓN}

$\mathbf{L}$ a lectura forma parte del proceso de comunicación, y como tal, es una de las destrezas que deben ser dominadas para poder comunicarse en un idioma extranjero, además es una herramienta para llegar al dominio del idioma estudiado. La importancia de la lectura es ampliamente avalada por diferentes autores (Benavides Cáceres, 2013), por ejemplo consideran que una persona para desarrollar de manera adecuada su formación debe ser lectora, puesto que el proceso de enseñanza y de aprendizaje en todos los ámbitos educativos, se establece mediado por la lectura y la escritura. Para Blanco (2010) "La lectura es la capacidad que tiene exclusivamente el hombre para transformar los contenidos recibidos en conocimiento". De acuerdo a Harmer (2008) existen tres razones para leer: en primer lugar muchos estudiantes necesitan tener la capacidad de leer en Inglés por sus carreras, por motivos de estudio o simplemente porque son ávidos lectores. La segunda razón, de acuerdo a este autor es que les ayuda en la adquisición del idioma, pues la lectura les provee de vocabulario y de

*Licenciada en Ciencias de la Educación Especialidad Inglés, Magister en Docencia e Investigación Educativa, Estudiante de Doctorado en Educación Superior.

${ }^{\dagger}$ Licenciada en Ciencias de la Educación Especialidad Inglés, Magister en Docencia e Investigación Educativa, Estudiante de Doctorado en Educación Superior. modelos de gramática. Por último, la tercera razón es que al leer obtienen modelos de cómo escribir tanto formal como informalmente.

En el caso de la destreza de la lectura en inglés como parte del aprendizaje de la lengua, los docentes tienden a tomarla como una actividad pasiva, que esquiva las estrategias activas y participativas, Ahora bien, es la lectura una actividad pasiva y personal? El objetivo de este estudio es demostrar que la lectura puede ser una actividad participativa en la que los estudiantes puedan interactuar en parejas o en grupos y que fomente la comunicación a través de estrategias activas y participativas.

En la concepción de los métodos activos y participativos prima la idea de "un aprendizaje de acción y recreación del conocimiento por los estudiantes mediante la actividad sistematizada" (Viñas Pérez, 2015). Lograr la participación activa de los estudiantes en su propio desarrollo nos lleva a un proceso interactivo. A través de este proceso el alumno puede tener una intervención reflexiva, consciente y sistematizada en la construcción y reconstrucción de sus conocimientos, habilidades y valores.

En este estudio se proponen cuatro estrategias específicas para convertir la lectura en un proceso activo y participativo que fomente la comunicación efectiva entre los estudiantes. Estas estrategias son: el mapa mental, el debate, la rejilla y el 
panel.

\section{DESARROLLO}

Partiendo de la premisa de que los tipos de estrategias activas y participativas que se utilizarán en una clase deben variar de acuerdo a la realidad del aula, ya que al decir de Javaloyes y Calderero "es preciso determinar la situación, tanto del alumno como del entorno en el que se va a desarrollar la tarea docente y las necesidades sociales existentes" (Bernardo, 2007) para que de esta manera todos se sientan parte importante del proceso de aprender, y para todos haya una actividad que se conecte con su propio estilo de aprendizaje y su tipo de inteligencia. Por lo tanto, la primera tarea del docente es evaluar la realidad de su grupo, el tema a tratar, las dimensiones del aula y el tiempo a utilizar antes de escoger la estrategia más apropiada. Otro factor a tener en cuenta es evitar que los estudiantes lean palabra por palabra y se detengan ante cada una que sea nueva para ellos, pues al decir de Scrivener (2011) no es esencial entender cada palabra sino lograr la decodificación del texto y la comprensión del mensaje del mismo. Por otro lado, y de acuerdo al decir de Ferris (2015), el uso de estas estrategias favorece el trabajo en equipo y genera actitudes de liderazgo entre los estudiantes, además fomenta el trabajo reflexivo y multidisciplinario y promueve la experiencia de respeto de las opiniones de todos los miembros del equipo y la toma de decisiones, lo cual se corrobora con la investigación de Coleman (2014) que sustenta que las actividades participativas generan liderazgo, y éste a su vez genera un sentido compartido de responsabilidad entre los miembros del equipo de trabajo. A continuación se hará un análisis de las estrategias sugeridas para potenciar la lectura comprensiva en Inglés: mapa mental, debate, rejilla y panel y se propondrá como utilizarlas en la lectura.

Los mapas mentales se van volviendo más populares conforme avanzan los años, según Blokehead (2015) “comparados con métodos tradicionales de lluvia de ideas, estudiar y tomar notas linealmente, el mapa mental es considerado del $15 \%$ $20 \%$ más efectivo para ayudar a la memoria y mejorar el aprendizaje". Se puede tomar los mapas mentales como Peña (2002) propone "una estrategia cognitiva, pues sirve para aprender, comprender, codificar y recordar la información orientada hacia un aprendizaje propuesto". Esta estrategia además permite a los alumnos ser conscientes de sus capacidades para pensar y para organizar la información que se convertirá después en aprendizaje. Es una herramienta que sirve para visualizar información y que por lo tanto permite a quienes lo utilizan a concentrarse en detalles concretos conservando una visión global. Esta estrategia tiene la particularidad de que favorece casi de manera instantánea la comprensión de la información.

Todo mapa mental debe cumplir con ciertos requerimientos, como los siguientes: El asunto o concepto que es motivo de nuestra atención o interés se expresa en una imagen central; los principales temas del asunto o concepto se desprenden de la imagen central de forma radial o ramificada; las ramas tienen una imagen y/o una palabra clave impresa sobre la línea asociada; los aspectos menos importantes también se representan como ramas adheridas a las ramas de nivel superior; y, las ramas forman una estructura conectada.

Un mapa mental no es más que la estructura compuesta de palabras, imágenes, fotografías y líneas con la intención de tener una organización visual de la información, lo cual permite por ejemplo, que del tema central tomado de un texto de lectura, se desprendan las ideas secundarias y otros detalles importantes que faciliten al lector resumir la información sintetizando lo más relevante de la misma y a la vez, le deja visualizar todo el contexto del texto leído.

En el caso de aprendizaje de una lengua, es una herramienta útil para actividades de comunicación oral y escrita, pues a partir de la información en un mapa mental previamente elaborado, se pueden extraer ideas para dar instrucciones, practicar conversaciones o hacer pequeños discursos, en el caso de la comunicación oral, y elaborar reportes, ensayos o historias en el caso de la comunicación escrita.

La lectura puede ser potencializada a través del uso de mapas mentales, pues mediante un mapa mental se pueden extraer las ideas principales del texto leído, las ideas secundarias o de sustento, también se puede incluir vocabulario nuevo y enlazarlo con el previamente adquirido, para de esta forma ampliar el mismo y asegurar la comprensión del texto leído. Esta actividad puede ser realizada en parejas o grupos muy pequeños (no más de tres estudiantes). El proceso inicia cuando el docente forma los grupos, luego les entrega o informa el texto a leer, los estudiantes empiezan a leer el texto y extraen la primera idea principal y la registra en un recuadro, luego, extraen las ideas de sustento de la principal y las registran en recuadros anexos al de la idea principal, siguen leyendo y toman la segunda idea principal para escribirla en otro recuadro, luego las ideas de sustento y así continúan hasta que hayan leído todo el texto y decodificado toda la información. A medida que avanzan en la lectura, irán encontrando vocabulario nuevo que pueden registrar anexos a las ideas de sustento. Al finalizar la lectura, les será fácil hacer un resumen de la misma.

Debates.- esta estrategia metodológica está ampliamente recomendada en la investigación de Fernández de Morgado (2016), quienes sugieren utilizarla durante y después de leer. Cuando se organizan actividades de debate, uno de los primeros pasos es informar, determinar y clarificar las reglas de participación. No basta con enlistar disposiciones de lo que se debe o no se debe hacer durante la actividad, sino que se debe hacer partícipes a los estudiantes del proceso de determinación de reglas, para lo cual, según Naidu (2011) él no es únicamente quien puede establecer reglas, sino que debe primar una política de equidad en el aula de clases. Se puede empezar informando de cuáles son los parámetros bajo los que se desarrollará el debate, pero dejando abierta la posibilidad de que esas reglas pueden cambiar de acuerdo a lo que decida el grupo. Una vez informados, los alumnos pueden expresar acuerdo o deseos de cambio de algún precepto, siempre y cuando se sustenten en una razón apropiada y que sea aceptada por los demás estudiantes. Una vez hechos los cambios sugeridos, el docente debe asegurarse de que las "reglas del juego" queden claras para todos los que van a participar del debate. Si se quiere que el debate sea una 
estrategia activa y participativa debe cumplir con la doble característica de que sea organizado por los estudiantes y que éstos participen de él. El éxito de esta técnica depende de la organización previa que el docente haga de todo el proceso del debate, anticipando detalles tales, como fijar las reglas de participación, determinar el tema, seleccionar los recursos y formar los equipos. El objetivo principal de esta técnica es la de "comprometer al mayor número posible de personas en la toma de decisiones" (Etxeberría, 2008).

Durante el debate, el rol del docente es el de moderador, quien, de acuerdo con Gutiérrez (2008), tiene dos claros objetivos, el primero es el de procurar que los integrantes de cada grupo se manifiesten y hablen libremente, de manera natural y espontánea; y el segundo objetivo es mantener al grupo enfocado en el tema del trabajo, sin salirse demasiado de él ante las opiniones generadas por ellos.

Aunque parezca más una técnica apropiada para desarrollar la destreza de comunicación oral, el debate también puede ser utilizado para el desarrollo de la destreza lectora. Es importante recordar que parte del proceso de lectura es la pre-lectura y la pos-lectura, y en estas partes del proceso, los estudiantes deberán expresar libremente ideas del tema que van a leer o ya leyeron, por lo tanto, el debate es una herramienta muy útil.

El proceso inicia con la explicación de las reglas de participación, y establecer el tiempo de duración de cada etapa, luego se forman los grupos, que pueden ser tres o cuatro, dependiendo del número de estudiantes, una vez formados los grupos el profesor entrega o informa el texto a leer y empieza la primera etapa. En esta primera etapa los estudiantes leen el texto y elaboran preguntas con respecto al tema leído, estas preguntas, que pueden ser cinco o más (dependiendo de la extensión del texto) deben ser de contenido y las respuestas deben estar en el texto; el profesor debe monitorear el proceso de la primera etapa asegurándose de que todos los alumnos tengan una idea clara sobre el texto y que las preguntas estén correctamente elaboradas y sean coherentes con la información leída. En la segunda etapa cada grupo hace preguntas al otro equipo, escucha, analiza y refuerza la respuesta de ser necesario; en esta etapa el docente hace las veces de moderador, asegurándose de que los alumnos participen en orden, que sean escuchados, que las respuestas sean correctas y retroalimentando de ser necesario.

Rejilla.- Esta técnica suele ser utilizada cuando se quiere que el grupo de estudiantes maneje cierta cantidad de información en poco tiempo, que la analice, sintetice y comparta en equipo. Además de permitir la comprensión de un texto rápidamente, de acuerdo a Barroso (2013), esta técnica sirve también para "Desarrollar la habilidad de síntesis, desarrollar la habilidad de presentar exposiciones, desarrollar la habilidad de trabajar en equipo, e incrementar el sentido de responsabilidad y solidaridad con los compañeros".

En un primer momento se conforman equipos, quienes leen y analizan un fragmento de la información que van a utilizar para construir un conocimiento o para entender un texto, cada miembro del grupo hace las veces de secretario, pues todos los miembros deben tomar notas, resumir y tener clara la información debatida. En un segundo momento, se forman equipos nuevos conformados por un estudiante de cada uno de los grupos previos, quienes explican a los demás el fragmento de la información que previamente han debatido en el primer equipo. En este momento de la rejilla, los grupos tienen la oportunidad de discutir la información en su totalidad. De ahí la importancia de que todos manejen con claridad el tema dado al inicio del trabajo.

Este método tiene la posibilidad de ser utilizado en la orientación inicial de los contenidos específicos o para complementar un primer momento de orientación presentado por el docente. Una de las ventajas de esta técnica es que ésta permite el intercambio de elementos esenciales del contenido que se aborda, incluso los estudiantes más destacados tienen la oportunidad de ayudar a quienes tienen dificultades en la comprensión de la lectura. Otra de las ventajas es que requiere de la participación individual responsable, sin la cual sería imposible el éxito del trabajo del segundo grupo, lo cual estimula a que los estudiantes hagan un esfuerzo personal para sintetizar la información.

Según Viñas una forma de optimizar la rejilla es "culminar con una plenaria donde cada equipo del segundo momento de discusión exponga los elementos más relevantes tratados previamente. Seleccionar al estudiante al azar, lo que el docente debe comunicar desde el inicio de la clase", de esta manera, los estudiantes participantes se concientizan sobre la importancia de su rol individual en el trabajo.

Panel.- En esta técnica, los estudiantes forman grupos para leer el documento o texto escogido por el docente, luego de un tiempo prudencial en que ya han leído, analizado y entendido el texto en cuestión, toman los roles de expertos que coordinados por un moderador, que es también parte del grupo, vierten opiniones sobre el tema. Durante el trabajo del equipo el moderador es quien se encarga de dirigir las rondas de preguntas y de pedir aclaraciones cuando lo considere conveniente además, según Boyd-Batstone (2013), también es parte del rol del moderador facilitar la discusión y asegurarse de que los panelistas contesten las preguntas. Los participantes no solo contestan preguntas, sino que pueden discutir en forma de diálogo sobre el tema en cuestión delante del grupo. Al finalizar, los estudiantes que hicieron las veces de observadores pueden intervenir con preguntas o comentarios sobre los temas tratados. Durante todo el proceso el docente observa, atiende y al final del panel interviene con la retroalimentación necesaria.

\section{Conclusiones}

- Los docentes de Inglés como lengua extranjera deben planificar clases que se ajusten con la dinámica que caracteriza a los estudiantes de este nuevo milenio, por tanto, las clases deben ser activas y participativas, permitiendo a los alumnos ser parte constante de su aprendizaje, y fomentando en ellos la interactuación no solo docente-estudiante, sino estudiante-estudiante.

- La lectura es una destreza receptiva del idioma Inglés, sin embargo esto no significa que deba ser una destreza pasiva. De hecho, el uso de estrategias activas y participativas no sólo potencia la lectura comprensiva, también permite a los alumnos valorar la eficacia del 
trabajo grupal, y de desarrollar a la par otras destrezas como la de resumir, preguntar y evaluar, y en cuanto a las destrezas comunicativas del idioma, el uso de estas estrategias fomenta la práctica de la comunicación oral y escrita.

- El debate, el panel, la rejilla y el mapa mental utilizados como estrategias activas y participativas para el desarrollo de la destreza lectora en Inglés, a través de una planificación eficaz realizada por el docente, se constituye en el tipo de actividad dinámica y flexible, lo cual, según los resultados de la investigación de Banguela, Nordarse y otros (2016), favorece el protagonismo estudiantil y se constituye en una forma novedosa y diferente de enseñanza aprendizaje, además genera confianza y liderazgo entre los estudiantes y favorece su participación constante, logrando con ello un aprendizaje significativo que se comprueba en la discusión y análisis de los textos leídos y decodificados.

\section{REFERENCIAS BIBLIOGRÁFICAS}

Barroso, Addy Beth; Barroso, R. y. P. G. (2013). Las dinámicas grupales y el proceso de aprendizaje.

Benavides Cáceres, Diana Raquel y Sierra Villamil, G. M. (2013). Estrategias didácticas para fomentar la lectura crítica desde la perspectiva de la transversalidad. REICE. Revista Electrónica Iberoamericana sobre Calidad, Eficacia y Cambio en Educación.

Bernardo, J.; Javaloyes, J. y. C. J. (2007). Cómo personalizar la educación. una solución de futuro.

Blanco, R. (2010). La lectura, un compromiso democrático. In La lectura, pages 13-20. Consejo Superior de Investigaciones Científicas, CSIC.

Blokehead, T. y Bravo, M. (2015). Mapas Mentales ;La Guía del Principiante Para Crear Mapas Mentales Paso a Paso! Babelcube Incorporated.

Boyd-Batstone, P. (2013). Helping English Language Learners Meet the Common Core: Assessment and Instructional Strategies $K-12$. Routledge.

Coleman, E. D. (2014). The Nature of Leadership: A Case Study of Distributed Leadership Amidst A Participative Change Effort. PhD thesis, The George Washington University.

Etxeberría, I. (2008). Intra-emprendizaje. Editorial Díaz de Santos, S.A.

Fernández de Morgado, Nelly Yiveline; Mayora Pernía, C. A. y. S. L. R. (2016). Pensamiento crítico y comprensión de la lectura en un curso de inglés como lengua extranjera. Íkala, revista de lenguaje y cultura, 21(1).

Ferris, H. (2015). The Use of Small Group Tutorials as an Educational Strategy in Medical Education. International Journal of Higher Education, 4(2).

Gutiérrez, J. (2008). Dinámica del grupo de discusión. Cuadernos metodológicos del Centro de Investigaciones Científicas, 41.

Harmer, J. (2008). How to teach english. ELT journal, 62(3):313-316.

Naidu, S. (2011). Teachers and the Policy Reform Agenda: Becoming Policy Aware, Policy Wise and Policy Active.
Peña, A.O.; Gómez, J. y. d. L. Á. (2002). Aprender con mapas mentales: Una estrategia para pensar y estudiar. Educación Hoy. Narcea.

Scrivener, J. (2011). Learning Teaching: The Essential Guide to English Language Teaching. Macmillan books for teachers. Macmillan Education.

Viñas Pérez, G. (2015). Los métodos participativos en una enseñanza desarrolladora. posibles soluciones a sus limitaciones. Revista Cubana de Educación Superior, (2):77-87. 